\title{
Infecciones como causa de ingreso urgente en adultos en un hospital terciario.
}

\author{
Miriam Saiz-Rodríguez ${ }^{1}$, Daniel Rafael Romero Palacian ${ }^{1}$, Francisco Abad-Santos ${ }^{1,2^{*}}$ \\ ${ }^{1}$ Servicio de Farmacología Clínica, Hospital Universitario de la Princesa, Instituto Teófilo Hernando, Universidad Autónoma de Madrid, Instituto de \\ Investigación Sanitaria de la Princesa (IP), Madrid, España. \\ ${ }^{2}$ Centro de Investigación Biomédica en Red de Enfermedades Hepáticas y Digestivas (CIBERehd), Instituto de Salud Carlos III, Madrid. \\ *Autor de correspondencia: Dr. Francisco Abad-Santos. Servicio de Farmacología Clínica, Hospital Universitario de la Princesa Diego de León 62. \\ 28006 Madrid (España) e-mail: francisco.abad@salud.madrid.org
}

\section{ABSTRACT}

OBJETIVO: Estudiar la prevalencia de las enfermedades infecciosas que son atendidas en el Servicio de Urgencias Hospitalario (SUH) y que requieren ingreso hospitalario, así como identificar los principales agentes causales y describir el tratamiento y evolución de los pacientes.

MÉTODO: Estudio observacional retrospectivo que evalúa todos los pacientes que requieren ingreso urgente en un hospital terciario durante el mes de Marzo de 2016. Se recogieron como variables: enfermedades concomitantes, agentes causales, cultivos realizados, resistencias antibióticas y tratamiento prescrito. Las infecciones se clasificaron en: urinarias, respiratorias, intraabdominales, infección de piel y partes blandas, neurológicas, y otras.

RESULTADOS: Ingresaron un total de 903 pacientes durante este periodo. De estos, se consideró que el 36,2\% podían tener una causa infecciosa. La edad media fue de 72,3 años y la estancia media en el hospital de 9,6 días. Las infecciones predominantes fueron respiratorias $(47,2 \%)$, urinarias (19\%) e intraabdominales $(18,1 \%)$. El $46,5 \%$ de las pruebas microbiológicas fueron positivas, siendo $E$. coli el principal agente causal (22,3\%). Los perfiles de sensibilidad de los aislados fueron similares a los esperados, excepto para $K$. pneumoniae ( $50 \%$ resistente a fluoroquinolonas y amoxicilina-clavulánico). Los antibióticos predominantemente prescritos fueron $\beta$-lactámicos $(51,2 \%)$ y fluoroquinolonas $(25,1 \%)$. La mortalidad fue del $7,3 \%$, siendo la edad y presentar criterios de sepsis factores de riesgo asociados.

CONCLUSIONES: Las infecciones son una de las principales causas de ingreso hospitalario y suponen un alto porcentaje de la atención sanitaria que se presta en el SUH. Nuestro estudio demuestra que la edad y los criterios de sepsis son factores cruciales asociados a la mortalidad de los pacientes. Este es el primer estudio que analiza cuantos ingresos a través del SUH se deben a una causa infecciosa.

Palabras clave: Infección bacteriana. Servicio de Urgencias. Ingreso hospitalario. Antibioterapia. Evolución hospitalaria. Recibido: 26, de octubre 2016; Accepted 22, de diciembre 2016; Published 11, de enero 2017.

Copyright: (c) 2017 Authors. This is an open-access article distributed under the terms of the Creative Commons Attribution License, which permits unrestricted use, distribution, and reproduction in any medium, provided the original author and source are credited.

Editor: Miriam Estébanez Muñoz

Citar como: Saiz-Rodriguez M., Romero Palacian DR., Abad-Santos F. Infecciones como causa de ingreso urgente en adultos en un hospital terciario. IBJ Clin Pharmacol 2017 1(1):e0004.

Fuentes de financiación: Miriam S.R. se encuentra cofinanciada por la Consejería de Educación, Juventud y Deporte de la Comunidad de Madrid y el Fondo Social Europeo.

Conflictos de intereses: Los autores no tienen conflictos de intereses relevantes a este artículo para divulgar. 


\section{Introducción}

A pesar de los grandes avances que se han producido en el conocimiento de las enfermedades infecciosas, estas continúan siendo una de las principales causas de morbimortalidad. En un estudio realizado en Estados Unidos se comprobó como la incidencia de infecciones aumentó considerablemente hasta constituir la tercera causa de muerte tras las enfermedades cardiovasculares y las neoplasias ${ }^{(1)}$.

Los procesos infecciosos suponen entre un 5\% y un $17 \%$ de las urgencias atendidas en los hospitales generales, así como una de las principales causas de ingreso hospitalario desde urgencias ${ }^{(2)}$. En un estudio español reciente, se vio como el $23,3 \%$ de los pacientes que llegan al Servicio de Urgencias Hospitalario (SUH) requieren ingreso por causa infecciosa ${ }^{(3)}$. Es en el SUH donde se realiza un primer diagnóstico y donde se inicia la antibioterapia, generalmente administrada de forma empírica ${ }^{(4)}$. A causa de esta elevada morbimortalidad se ha comenzado a poner en perspectiva la importancia de administrar el tratamiento antibiótico adecuado en cuanto el paciente llega al SUH ${ }^{(5)}$. Es imprescindible conocer cuáles son los agentes causales de las infecciones graves, que son las que requieren ingreso hospitalario. En el entorno hospitalario las infecciones que por su gravedad y frecuencia constituyen los problemas infecciosos más frecuentes son las neumonías, las infecciones del tracto urinario y las infecciones del sistema nervioso central ${ }^{(6)}$.

Por ello, el objetivo de este estudio es analizar la prevalencia de las enfermedades infecciosas graves que son atendidas en el SUH y que finalmente requieren ingreso hospitalario, así como identificar los principales agentes causales de las mismas y describir el tratamiento y evolución de los pacientes.

\section{Método}

Se trata de un estudio observacional retrospectivo aprobado por el Comité de Ética de la Investigación del Hospital Universitario de la Princesa. El Hospital Universitario de la Princesa es un hospital terciario de la Comunidad de Madrid, ubicado en el Barrio de Salamanca de Madrid. Su cartera de servicios cubre todas las especialidades médicas y quirúrgicas excepto la de Obstetricia y Ginecología y Pediatría. La población asignada al centro incluye los Centros de Salud colindantes, con aproximadamente 310.000 habitantes en su conjunto (Figura 1b).

Se revisaron todos los pacientes que ingresaron a través del Servicio de Urgencias durante el mes de Marzo de 2016. Los casos a estudiar fueron todos aquellos en los que el motivo de ingreso fuera una sospecha clínica de infección bacteriana. Se realizó un seguimiento de la evolución de los pacientes hasta el alta hospitalaria o fallecimiento para comprobar si se confirma la sospecha de infección bacteriana, si se identifica el microorganismo responsable y cuál es su perfil de resistencias a antimicrobianos. Para mantener la confidencialidad de los pacientes no se recogió ningún dato identificativo y a cada paciente se le asignó un código de números correlativos.
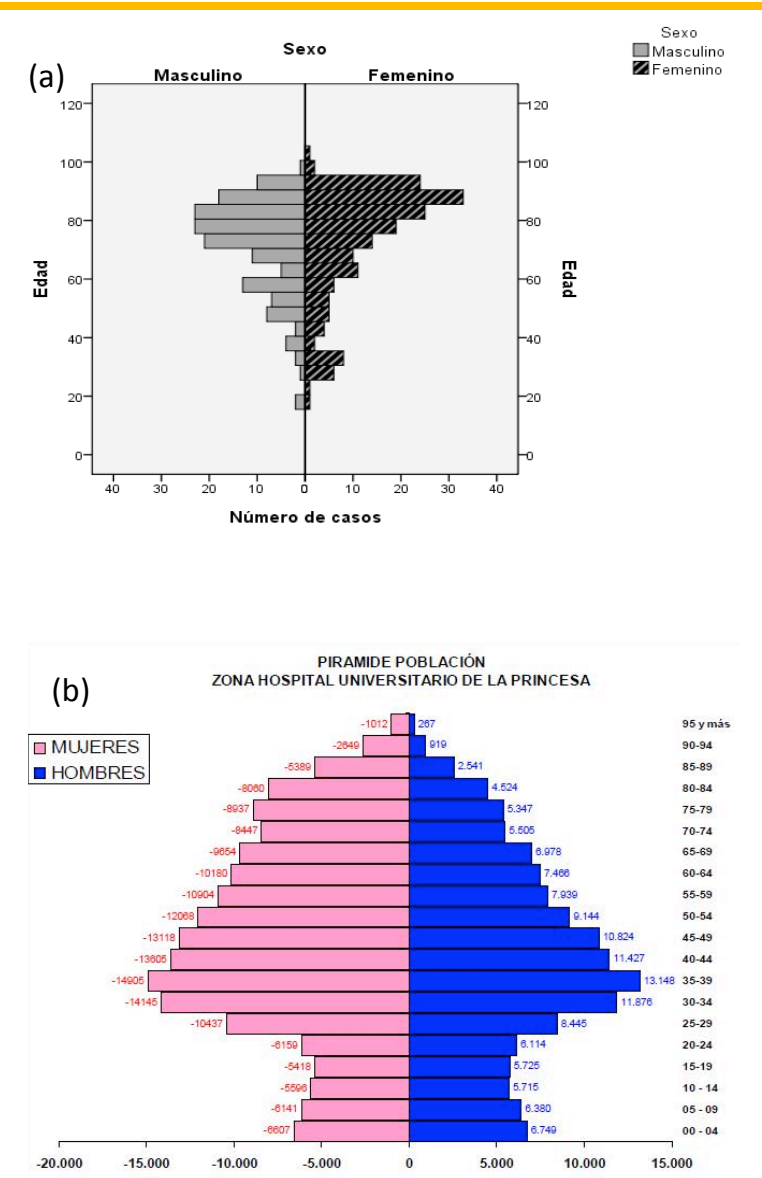

Figura 1. Pirámide poblacional de los pacientes ingresados a través del Servicio de Urgencias en este estudio (a) en comparación con la población asignada al Hospital Universitario de la Princesa (b) (Datos de 2013, últimos disponibles en la página web del Hospital de la Princesa).

Se recogieron las variables demográficas (sexo, edad), el motivo del ingreso, el tipo de infección diagnosticada, la presencia o no de criterios de sepsis, la comorbilidad (diabetes, cardiopatías, enfermedad pulmonar obstructiva crónica (EPOC), hepatopatía crónica, insuficiencia renal crónica, infección por VIH, neoplasias), los factores de riesgo de multirresistencia (portador de sonda vesical o catéter, antibiótico previo, procedencia de 
una institución de cuidados o residencia, infección nosocomial), las pruebas microbiológicas obtenidas (urocultivos, hemocultivos, coprocultivos, esputo, secreciones, líquidos y exudados), el agente infeccioso identificado, las co-infecciones, el resultado del antibiograma, el antibiótico prescrito en urgencias y el definitivo durante la hospitalización, los días de ingreso y si se procedió a darle el alta o falleció. Para la variable antibiótico previo se consideró un periodo de una semana previo a la llegada a Urgencias, pues este supone un ciclo completo de la administración de antibióticos.El diagnóstico de sepsis se basó en el criterio del médico responsable del paciente.

Se consideraron microorganismos multirresistentes a aquellos que fueron resistentes a una o más de una familia o grupo de antibióticos de uso habitual, con cierta relevancia clínica y epidemiológica ${ }^{(7)}$. Este término se usa sobre todo para bacterias ligadas al ámbito sanitario que han desarrollado mecanismos de resistencia y que son capaces de producir brotes ${ }^{(8)}$. Los microorganismos se consideraron sensibles o resistentes en base a la guía EUCAST en el caso de tener esta punto de corte para el antibiótico en estudio, si no fuera así se clasificó en base a la guía CLSI.

Las infecciones que se consideraron nosocomiales se corresponden a aquellas contraídas por un paciente durante su tratamiento en un hospital u otro centro sanitario y que dicho paciente no padecía en el momento de su ingreso. Por otra parte, se contemplaron las infecciones asociadas al cuidado sanitario siendo estas asociadas a aquellos pacientes que provenían de una institución de cuidados o residencia de ancianos.

Las infecciones se clasificaron en: urinarias (pielonefritis aguda, infecciones urinarias de vías bajas, prostatitis, infecciones urinarias en paciente con sonda vesical), respiratorias de vías bajas (bronquitis aguda, exacerbación infecciosa de una EPOC, bronquiectasias infectadas, neumonía, absceso pulmonar), intraabdominales (infección hepatobiliar, apendicitis, diverticulitis, peritonitis), infección de piel y partes blandas (necrosante, no necrosante, infección del pie diabético, infección de úlceras por presión), neurológicas (meningitis, encefalitis), y otras (síndrome gripal, sepsis sin filiar, síndromes febriles, bacteriemia, abscesos, pericarditis, oftalmológicas, etc). El tipo de infección se defino en base al diagnóstico final del informe de alta hospitalaria.

El análisis estadístico se llevó a cabo con el paquete SPSS statistics 19.0. Se utilizó la frecuencia absoluta y relativa para describir las variables cualitativas y la media con desviación estándar para las variables cuantitati- vas. Para las principales variables se calculó el intervalo de confianza del 95\%. Se utilizó la chi-cuadrado para la comparación de las variables cualitativas y la t de Student para el análisis de las variables cuantitativas o análisis de la varianza (ANOVA) si eran más de dos grupos. Se utilizó una regresión logística para analizar la relación entre distintos factores y la mortalidad, incluyéndose todas aquellas variables que consideramos con relevancia clínica: sexo, edad, tipo de infección e ingresar con criterios de sepsis. Se consideró estadísticamente significativo un valor de $\mathrm{p}<0,05$.

\section{Resultados}

Durante el mes de marzo de 2016, ingresaron 1342 pacientes en el Hospital de la Princesa. De estos, 903 procedían del Servicio de Urgencias; esto supuso 29,1 ingresos urgentes diarios de media.

De los ingresos procedentes del SUH, 328 se debían a una posible causa infecciosa. Durante ese mismo mes 9 pacientes volvieron a ingresar por causa infecciosa, de modo que se analizaron 337 ingresos en total. Por lo tanto el 36,2\% (IC95\%: 32,9\%-39,1\%) del total de ingresos procedentes de urgencias se deben a posible causa infecciosa. Los principales tipos de infección fueron respiratorias $(47,2 \%)$, urinarias $(19 \%)$ e intraabdominales $(18,1 \%)$ (Tabla 1$)$.

Tabla 1. Tipo de infección en los pacientes que ingresaron a través del Servicio de Urgencias por causa infecciosa.

\begin{tabular}{|c|c|c|}
\hline $\begin{array}{c}\text { TIPO DE } \\
\text { INFECCIÓN }\end{array}$ & $\begin{array}{l}\text { NÚMERO DE } \\
\text { CASOS }\end{array}$ & $\begin{array}{l}\text { PORCENTAJE DEL } \\
\text { TOTAL (IC 95\%) }\end{array}$ \\
\hline Respiratoria & 159 & $\begin{array}{c}47,2 \% \\
(41,7 \%-52,3 \%)\end{array}$ \\
\hline Urinaria & 64 & $19,0 \%(15 \%-23 \%)$ \\
\hline Intraabdominal & 61 & $18,1 \%(14 \%-22 \%)$ \\
\hline $\begin{array}{l}\text { Infecciones de piel } \\
\text { y partes blandas }\end{array}$ & 12 & $3,6 \%(1,6 \%-5,4 \%)$ \\
\hline Meningitis & 3 & $0,9 \%(0 \%-1,9 \%)$ \\
\hline $\operatorname{Mixtas}^{1}$ & 10 & $3,0 \%(1,2 \%-4,6 \%)$ \\
\hline Otras $^{2}$ & 28 & $\begin{array}{c}8,3 \%(5,4 \%- \\
11,2 \%)\end{array}$ \\
\hline $\begin{array}{l}\text { Total } \\
1 \text { Las infecciones mixt } \\
\text { la vez una infección } \\
\text { ingreso como fiebre } \\
\text { fracaso en paciente } \\
\text { carditis, monoartritis }\end{array}$ & $\begin{array}{l}337 \\
\text { luyen pacient } \\
\text { ratoria y urin } \\
\text { co, sepsis sin } \\
\text { positivos, bac } \\
\text { oftalmitis. }\end{array}$ & $\begin{array}{l}\text { los que se registró a } \\
{ }^{2} \text { Incluye motivos de } \\
\text { r, síndromes febriles, } \\
\text { emia, abscesos, peri- }\end{array}$ \\
\hline
\end{tabular}

El servicio que más pacientes ingresó fue el de Medicina Interna $(29,4 \%)$, seguido de Neumología (24\%), Digestivo $(9,5 \%)$, Infecciosas $(8,6 \%)$ y Cirugía General $(5,9 \%)$.

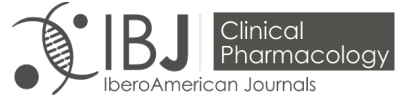

www.ibjournals.com 
El $46 \%$ de los pacientes que ingresaron por causa infecciosa fueron varones $(n=151)$ y el $54 \%$ mujeres $(n=177)$. La media de edad de los pacientes fue de $72,3 \pm 18,0$ años, con un rango de [18-101] (Figura 1). La edad media de los varones fue de 71,5 $\pm 16,2$ años, y de las mujeres 73,2 $\pm 19,4$ años $(p=0,385)$. La media de edad de los pacientes varía según el tipo de infección (Tabla 2), siendo la más alta para las infecciones urinarias $(75,5$ años) y la más baja para las infecciones neurológicas (43,5 años) pero esta diferencia no alcanza la significación estadística $(\mathrm{p}=0,068)$. El rango etario con mayor número de casos fue el de 70-89 años (53\%). En todos los rangos de edad, las infecciones más frecuentes son las respiratorias pero destaca especialmente en el grupo de 70-89 años, que además tienen menos infecciones intraabdominales. Tanto hombres como mujeres padecieron principalmente infecciones respiratorias y no hubo diferencias significativas entre ellos en el tipo de infección (Tabla 2).

Estratificando los pacientes en base al lugar de adquisición de la infección: comunitaria $(n=305 ; 92,9 \%)$, asociada al cuidado sanitario $(\mathrm{n}=16 ; 4,9 \%)$ o nosocomial $(\mathrm{n}=7 ; 2,1 \%)$, se observó una edad media más alta en aquellos provenientes de una institución $(85,1 \pm 8.1$, $\mathrm{p}=0,009)$ en comparación con los pacientes con infecciones comunitarias $(71,5 \pm 18.2)$ y nosocomiales $(81,6$ $\pm 6,8$ ). En las infecciones comunitarias y nosocomiales el $47,2 \%$ y $57,1 \%$ fueron varones, respectivamente. Sin embargo, estos solo representaron el 18,7\% de las infecciones asociadas al cuidado sanitario. Las infecciones más comunes en las asociadas al cuidado sanitario y comunitarias fueron las respiratorias $(47,4 \%$ y $56,2 \%$, respectivamente); en las infecciones nosocomiales las más frecuentes fueron las urinarias $(42,8 \%)$. Todos los pacientes provenientes de una institución residencial o con infecciones nosocomiales padecían algún tipo de comorbilidad.

Un total de 292 (89\%) pacientes presentaban comorbilidades como hipertensión arterial, diabetes mellitus, cardiopatías, neoplasias o EPOC (Tabla 3). Se encontró asociación estadísticamente significativa entre la presencia de EPOC y las infecciones respiratorias $(\mathrm{p}<0,001)$, la uropatía y las infecciones urinarias $(\mathrm{p}=0,003)$, el estado de inmunosupresión y las infecciones mixtas $(p<0,001)$ y el ser portador de catéter y las infecciones urinarias $(\mathrm{p}=0,001)$ (Tabla 3$)$.

El 15,1\% del total de pacientes cumplían criterios de sepsis a su llegada al SUH, lo que se corresponde con el $11,9 \%$ de las infecciones respiratorias, $21,9 \%$ de las urinarias, $14,8 \%$ de las intraabdominales, el 8,3 de las infecciones de piel y partes blandas y el $28,6 \%$ de otras infecciones $(p=0,116)$.

En un 87,5\% $(\mathrm{n}=295)$ de los pacientes se realizó algún estudio microbiológico (Tabla 4). Los tres cultivos microbiológicos más frecuentes fueron el urocultivo (51,3\% de los pacientes), el hemocultivo (49,3\%) y el exudado $(48,1 \%)$. Destaca que en las infecciones respiratorias la prueba microbiológica más utilizada fue el cultivo de exudados nasofaríngeos $(n=109,68,5 \%)$ y que en casi la totalidad de las infecciones urinarias se realizaron urocultivos ( $\mathrm{n}=62,96,9 \%$ ) (Tabla 4). Los cultivos sólo fueron positivos para algún microorganismo en el $46,5 \%$ de los casos $(n=137)$. En las infecciones respiratorias el porcentaje de aislados positivos fue del 27,7\% $(n=44)$, en las urinarias del $64,1 \%(n=41)$ y en las intraabdominales del 42,6\% $(n=26)(p=0,002)$.

En las pruebas microbiológicas se logró identificar un total de 57 microorganismos, de los cuales el más frecuente fue Escherichia coli (22,3\%), seguido de Pseu-

Tabla 2. Tipos de infección según el rango de edad y el sexo de los pacientes.

\begin{tabular}{|c|c|c|c|c|c|c|c|c|}
\hline & IR (n=159) & ITU $(n=64)$ & $\underset{\substack{\text { IIA } \\
(n 1)}}{ }$ & $\begin{array}{l}\text { IPPB } \\
(n=12)\end{array}$ & $\begin{array}{l}\text { IN } \\
(n=3)\end{array}$ & $\begin{array}{l}\text { Mixtas } \\
(n=10)\end{array}$ & $\begin{array}{l}\text { Otras } \\
(n=28)\end{array}$ & $\begin{array}{l}\text { Total } \\
(n=337)\end{array}$ \\
\hline $\begin{array}{l}\text { Edad media } \\
\text { (DE) }\end{array}$ & $74,4(16,8)$ & $75,5(15,6)$ & $67,2(21,2)$ & $62(26,4)$ & $43,5(0,7)$ & $75,2(9)$ & $71,5(16,2)$ & \\
\hline \multicolumn{9}{|c|}{ Grupo de edad ${ }^{1}: \mathrm{n}(\%)$} \\
\hline $18-29$ años & $4(40,0)$ & $0(0)$ & $4(40,0)$ & $2(20,0)$ & $0(0)$ & $0(0)$ & $0(0)$ & $10(3)$ \\
\hline 30-49 años & $11(35,5)$ & $4(12,9)$ & $9(29,0)$ & $2(6,5)$ & $2(6,5)$ & $0(0)$ & $3(9,7)$ & $31(9,5)$ \\
\hline 50-69 años & $27(39,7)$ & $12(17,6)$ & $15(22,1)$ & $3(4,4)$ & $0(0)$ & $3(4,4)$ & $8(11,8)$ & $68(20,7)$ \\
\hline 70-89 años & $92(52,9)$ & $35(20,1)$ & $24(13,8)$ & $3(1,7)$ & $0(0)$ & $7(4,0)$ & $13(7,5)$ & $174(53)$ \\
\hline$>90$ años & $20(44,4)$ & $10(22,2)$ & $9(20,0)$ & $2(4,4)$ & $0(0)$ & $0(0)$ & $4(8,9)$ & $45(13,7)$ \\
\hline \multicolumn{9}{|l|}{ Sexo: n (\%) } \\
\hline Hombre & $73(48,3)$ & $28(18,5)$ & $27(17,9)$ & $7(4,6)$ & $1(0,7)$ & $3(2)$ & $12(7,9)$ & $151(46)$ \\
\hline Mujer & $81(45,8)$ & $33(18,6)$ & $34(19,2)$ & $5(2,8)$ & $1(0,6)$ & $7(4)$ & $16(9)$ & $177(54)$ \\
\hline
\end{tabular}

IR: infecciones respiratorias; ITU: infecciones del tracto urinario; IIA: infecciones intraabdominales; IPPB: infecciones de piel y partes blandas; IN: infecciones neurológicas. ${ }^{1}$ Se contabilizan únicamente los primeros ingresos para evitar el artefacto de los reingresos. 
Tabla 3. Enfermedades concomitantes de los pacientes.

\begin{tabular}{|c|c|c|c|c|}
\hline Comorbilidad & $\begin{array}{c}\text { Total }(n=328) \mathrm{N} \\
(\%)\end{array}$ & $\begin{array}{l}\text { Infecciones respir- } \\
\text { atorias }^{1}(n=154) n \\
(\%)\end{array}$ & $\begin{array}{l}\text { Infecciones uri- } \\
\text { narias }^{2}(n=61) n \\
(\%)\end{array}$ & $\begin{array}{c}\text { Infecciones in- } \\
\text { traabdominales }(n=61) \\
n(\%)\end{array}$ \\
\hline Hipertensión arterial & $140(42,7)$ & $62(40,3)$ & $28(45,9)$ & $28(45,9)$ \\
\hline Dislipemia & $86(26,2)$ & $38(24,7)$ & $19(31,1)$ & $15(24,6)$ \\
\hline Diabetes mellitus & $69(21)$ & $32(20,8)$ & $14(23)$ & $13(21,3)$ \\
\hline Cardiopatía & $68(20,7)$ & $40(26)$ & $9(14,8)$ & $9(14,8)$ \\
\hline Patología tumoral & $61(18,6)$ & $25(16,2)$ & $14(23)$ & $8(13,1)$ \\
\hline EPOC & $56(17,1)$ & $44(28,6)^{*}$ & $4(6,6)$ & $3(4,9)$ \\
\hline Uropatía & $40(12,2)$ & $14(9,1)$ & $16(26,2)^{*}$ & $2(3,3)$ \\
\hline Inmunosupresión & $35(10,7)$ & $12(7,8)$ & $5(8,2)$ & $7(11,5)$ \\
\hline Hepatopatía & $24(7,3)$ & $7(4,5)$ & $4(6,6)$ & $5(8,2)$ \\
\hline Portador de catéter & $17(5,2)$ & $3(1,9)$ & $10(16,4)^{*}$ & - \\
\hline Asma & $13(4)$ & $12(7,8)$ & - & $1(1,6)$ \\
\hline EPID & $3(0,9)$ & $1(0,6)$ & $1(1,6)$ & - \\
\hline Fibrosis quística & $2(0,6)$ & $2(1,3)$ & - & - \\
\hline
\end{tabular}

domonas aeruginosa (9,1\%), Staphyloccocus aureus $(6,4 \%)$, Klebsiella pneumoniae $(5,9 \%)$, Enterococcus faecium (5\%) y Haemophilus influenzae (5\%). En total, en $200(59,3 \%)$ pacientes no se pudo identificar el agente causante de la infección, bien porque no se realizaron pruebas microbiológicas $(n=42)$ o bien porque estas fueron negativas $(n=158)$. En las infecciones respiratorias, se aisló principalmente $H$. influenzae $(25 \%), P$. aeruginosa $(11,4 \%)$ y E. coli $(6,8 \%)$. En las infecciones urinarias los microorganismos mayoritariamente aislados fueron $E$. coli $(48,8 \%), K$. pneumoniae $(12,2 \%)$ y $P$. aeruginosa $(9,7 \%)$. En las infecciones intraabdominales se aisló principalmente E. coli $(34,6 \%)$, Clostridium difficile toxigénico $(15,4 \%)$, Enterococcus faecalis (7,7\%) y E. faecium $(7,7 \%)$.

En el $13,9 \%(n=47)$ de las infecciones se produjo una co-infección de diferentes microorganismos bacterianos. Además, en $34(21,4 \%)$ y $20(12,6 \%)$ pacientes con infección respiratoria se detectó la presencia del virus Influenza A e Influenza $B$, respectivamente. De estos 54 casos, 24 fueron co-infecciones bacterianas. Al alta uno de los pacientes con sospecha clínica de infección bacteriana sólo padecía infección vírica.

Se analizaron las resistencias de los microorganismos más prevalentes. Las cepas de $E$. coli fueron resistentes a $\beta$-lactámicos de amplio espectro en un $14 \%$, a ampicilina en un $70 \%$ y a quinolonas en un $30 \%$, pero todas fueron sensibles a carbapenémicos, aminoglucósidos, nitrofurantoina y fosfomicina. El $50 \%$ de las cepas de $K$. pneumoniae fueron resistentes a fluoroquinolonas y amoxicilina-ácido clavulánico y el $60 \%$ a fosfomicina. El $60 \%$ de las cepas de $P$. aeruginosa fue resistente a tobramicina, el $45 \%$ a fluoroquinolonas y el $26 \%$ a carbapenémicos. $S$. aureus fue resistente a meticilina en un $21 \%$ y a fluoroquinolonas en un $20 \%$. El $60 \%$ de las cepas de E. faecium fueron resistentes a ampicilina y fluoroquinolonas. El $30 \%$ de las cepas de E. faecalis fueron resistentes a fluoroquinolonas. No hubo ningún enterococo resistente a vancomicina (Tabla 5).

Respecto a la prescripción de antibióticos en el SUH la familia predominante fueron los $\beta$-lactámicos $(51,2 \%)$, seguidos por las fluoroquinolonas $(25,1 \%)$, porcentajes similares a la administración definitiva durante el ingreso $(51 \%$ y $29 \%$, respectivamente). Sin embargo, los antibióticos mayoritariamente prescritos varían según el tipo de infección: en las infecciones respiratorias se pautó principalmente levofloxacino $(34,7 \%)$, amoxicilina-ácido clavulánico $(17,3 \%)$ y moxifloxacino $(6,6 \%)$; en las urinarias ceftriaxona $(35,5 \%)$, meropenem $(13,1 \%)$ y ertapenem $(6,6 \%)$ y en las intraabdominales piperacilina-tazobactam $(26,7 \%)$, carbapenémicos (ertapenem y meropenem, 17,8\%) y amoxicilina-ácido clavulánico $(11,9 \%)$. Finalmente, en un $2,6 \%$ de los casos no se administró tratamiento antibiótico, presumiblemente por no haberse confirmado la sospecha de causa bacteriana en la infección. Sólo en el 18,7\% (n=63) se pudo evaluar la adecuación del antibiótico prescrito en urgencias por ajuste al antibiograma y en el $92 \%$ de ellos la prescripción empírica fue acertada.

El 7,3\% (IC95\%=5,9\%-8,7\%) de los pacientes fallecieron, presentando una edad media de $82,1 \pm 11,2$ años, superior a la del resto de los pacientes $(\mathrm{p}=0,006)$. 
Tabla 4. Cultivos realizados a los pacientes que ingresan por sospecha de infección.

\begin{tabular}{lcccc}
\hline Tipo de cultivos & $\begin{array}{c}\text { Número de pruebas } \\
\text { totales (N=337) }\end{array}$ & $\begin{array}{c}\text { Número de pruebas } \\
\text { en infecciones respi- } \\
\text { ratorias } \mathbf{( n = 1 5 9 )}\end{array}$ & $\begin{array}{c}\text { Número de pruebas } \\
\text { en infecciones urina- } \\
\text { rias (n=64) }\end{array}$ & $\begin{array}{c}\text { Número de pruebas en in- } \\
\text { fecciones intraabdominales } \\
\text { (n=61) }\end{array}$ \\
\hline Urocultivo & $173(51,3 \%)$ & $63(39,6 \%)$ & $62(96,9 \%)^{*}$ & $23(37,7 \%)$ \\
Hemocultivo & $166(49,3 \%)$ & $74(47,8 \%)$ & $39(60,9 \%)$ & $25(41 \%)$ \\
Esputo & $80(23,7 \%)$ & $66(41,5 \%)^{*}$ & $6(9,4 \%)$ & - \\
Secreción & $39(11,6 \%)$ & $11(6,9 \%)^{*}$ & $3(4,7 \%)$ & $14(22,9 \%)$ \\
Líquidos estériles & $15(4,5 \%)$ & $9(5,7 \%)$ & $1(1,6 \%)$ & - \\
Exudados & $161(47,8 \%)$ & $109(68,5 \%)^{*}$ & $14(21,9 \%)$ & $13(21,3 \%)$ \\
Coprocultivo & $46(13,6 \%)$ & $16(10,1 \%)$ & $6(9,4 \%)$ & $14(22,9 \%)$ \\
\hline Total & $\mathbf{6 8 0}$ & $\mathbf{3 4 8}$ & $\mathbf{1 3 1}$ & $\mathbf{8 9}$
\end{tabular}

${ }^{1}$ Incluye muestras de líquido cefalorraquídeo $(n=4)$ y líquido pleural $(n=11)$, se encontró asociación significativa con las meningitis $(p=0,000)$. $* \mathrm{p}<0.05$.

El porcentaje de muertes atribuibles a causa infecciosa fue del $66,7 \%$, según el criterio clínico recogido en el informe de alta.

La estancia media en el hospital fue de 9,6 días $\pm 8,4$, siendo más alta en infecciones de piel y partes blandas y meningitis $(\mathrm{p}=0,08)$ (Tabla 5). En los pacientes que presentaban co-infecciones la estancia media en el hospital aumenta hasta los 15,2 días, con asociación estadísticamente significativa $(\mathrm{p}=0,004)$. La evolución de los pacientes fue diferente según el tipo de infección por la que ingresaran, siendo la mortalidad más alta en las infecciones mixtas $(20 \%)$ y en otras infecciones $(17,9 \%)$, aunque no alcanza la significación estadística $(\mathrm{p}=0,138)$ (Tabla 6).

Cuando analizamos conjuntamente todos los factores de riesgo que pueden influir sobre la mortalidad observamos que la edad influye sobre la misma con una asociación significativa $(p=0,01)$, incrementando un $5 \%$ el riesgo por cada incremento de un año de edad. A su vez, el hecho de ingresar con criterios de sepsis también se asocia significativamente a la mortalidad $(p=0,03)$, aumentando alrededor de 3 veces más el riesgo de fallecimiento (Tabla 7).

\section{Discusión}

Los Servicios de Urgencias son muy útiles para la vigilancia epidemiológica porque aportan una gran cantidad de información sobre diversas enfermedades, entre ellas las causadas por agentes infecciosos. Por esto deben considerarse piezas fundamentales para la detección precoz, tratamiento y prevención de las infecciones emergentes y reemergentes $^{(9)}$.

En nuestro estudio, un tercio de los ingresos que se tramitan a través del SUH se deben a una posible causa infecciosa. Este porcentaje es más elevado que el encon- trado en otro estudio $(9,7 \%)$ que analiza los ingresos hospitalarios por enfermedades infecciosas de 1999 a $2003^{(10)}$, lo que se puede explicar por la edad de los pacientes que se atienden en el Hospital de la Princesa, en el que sólo se atienden adultos y principalmente de una zona con la población considerablemente envejecida (la edad media de nuestro estudio es de 72 años frente a 38 años en el otro estudio). También puede influir la fecha de realización del estudio (Marzo de 2016 frente a 5 años desde 1999 a 2003).

Las enfermedades infecciosas se asociaban en el pasado a los pacientes jóvenes con alteraciones inmunitarias $^{(3)}$; sin embargo, en el momento actual la gran mayoría de las infecciones las padecen los pacientes más ancianos $^{(11)}$. Los cambios fisiológicos e inmunológicos que ocurren con la edad complican la identificación y el diagnóstico de una infección en las personas de edad avanzada $^{(11,12)}$. Por tanto, los ancianos tienen mayor riesgo de padecer una enfermedad infecciosa y de que esta pase desapercibida o sea mal diagnosticada ${ }^{(12)}$. Las infecciones bacterianas agudas que afectan a los ancianos suponen un mayor coste sanitario ya que deben permanecer más días ingresados y el tratamiento se complica por la presencia de comorbilidades ${ }^{(12)}$.

En el estudio INFURG-SEMES, realizado en el año 2013, se refleja la epidemiología de las infecciones atendidas en el SUH de los hospitales españoles. La prevalencia de enfermedades infecciosas fue del 14,3\% (4,6\% respiratoria, 3,2\% urinaria, $2,1 \%$ otorrinolaringológica, $1,6 \%$ infecciones de piel y partes blandas y $2,8 \%$ otras); el $39,8 \%$ de los pacientes presentaban enfermedades asociadas como la diabetes mellitus, cardiopatía o EPOC; y el $6,2 \%$ cumplían criterios de sepsis a su llegada a urgencias. Respecto al manejo, no se realizó ningún estudio microbiológico en el $56,7 \%$ de los pacientes, y la amoxicilina-ácido clavulánico fue el antibiótico más 
Tabla 5. Resultados de sensibilidad de los principales aislados.

\begin{tabular}{|c|c|c|c|c|c|}
\hline Microorganismo & Antibióticos & Sensible & Intermedio & Resistente & $\mathrm{N}^{*}$ \\
\hline \multirow[t]{11}{*}{ E. coli $(n=49)$} & Amoxicilina/Clavulánico & $32(71,1 \%)$ & - & $13(28,9 \%)$ & 45 \\
\hline & Ampicilina & $13(31,7 \%)$ & - & $28(68,3 \%)$ & 41 \\
\hline & Cefuroxima & $31(100 \%)$ & - & - & 31 \\
\hline & Ciprofloxacina & $33(67,3 \%)$ & - & $16(32,7 \%)$ & 49 \\
\hline & Cotrimoxazol & $26(56,5 \%)$ & - & $20(43,5 \%)$ & 46 \\
\hline & Ertapenem & $20(100 \%)$ & - & - & 20 \\
\hline & Fosfomicina & $33(97,1 \%)$ & - & $1(2,9 \%)$ & 34 \\
\hline & Gentamicina & $42(85,7 \%)$ & $1(2 \%)$ & $6(12,2 \%)$ & 49 \\
\hline & Nitrofurantoina & $30(100 \%)$ & - & - & 30 \\
\hline & Norfloxacino & $15(53,6 \%)$ & $2(7,1 \%)$ & $11(39,3 \%)$ & 28 \\
\hline & Piperacilina/tazobactam & $11(57,9 \%)$ & $2(10,5 \%)$ & $6(31,6 \%)$ & 19 \\
\hline \multirow{8}{*}{$\begin{array}{l}\text { P. aeruginosa } \\
(n=20)\end{array}$} & Aztreonam & $2(20 \%)$ & $7(70 \%)$ & $1(10 \%)$ & 10 \\
\hline & Ceftazidima & $13(81,3 \%)$ & - & $3(18,8 \%)$ & 16 \\
\hline & Ciprofloxacino & $8(47,1 \%)$ & $1(5,9 \%)$ & $8(47,1 \%)$ & 17 \\
\hline & Colistina & $8(72,7 \%)$ & $2(18,2 \%)$ & $1(9,1 \%)$ & 11 \\
\hline & Imipenem & $12(70,6 \%)$ & - & $5(29,4 \%)$ & 17 \\
\hline & Meropenem & $12(70,6 \%)$ & $1(5,9 \%)$ & $4(23,5 \%)$ & 17 \\
\hline & Piperacilina/tazobactam & $13(76,5 \%)$ & $3(17,6 \%)$ & $1(5,9 \%)$ & 17 \\
\hline & Tobramicina & $4(40 \%)$ & - & $6(60 \%)$ & 10 \\
\hline \multirow{11}{*}{$\begin{array}{l}\text { S. aureus } \\
(n=14)\end{array}$} & Amoxicilina/clavulánico & $4(100 \%)$ & - & - & 4 \\
\hline & Ciprofloxacina & $10(76,9 \%)$ & - & $3(23,1 \%)$ & 13 \\
\hline & Clindamicina & $7(87,5 \%)$ & - & $1(12,5 \%)$ & 8 \\
\hline & Cloxacilina & $11(78,6 \%)$ & - & $3(21,4 \%)$ & 14 \\
\hline & Cotrimoxazol & $14(100 \%)$ & - & - & 14 \\
\hline & Fosfomicina & $6(100 \%)$ & - & - & 6 \\
\hline & Gentamicina & $11(91,7 \%)$ & - & $1(8,3 \%)$ & 12 \\
\hline & Levofloxacino & $10(76,9 \%)$ & - & $3(23,1 \%)$ & 13 \\
\hline & Linezolid & $7(100 \%)$ & - & - & 7 \\
\hline & Teicoplanina & $4(100 \%)$ & - & - & 4 \\
\hline & Vancomicina & $7(100 \%)$ & - & - & 7 \\
\hline \multirow{9}{*}{$\begin{array}{l}\text { K. pneumoniae } \\
(n=13)\end{array}$} & Amoxicilina/clavulánico & $5(50 \%)$ & - & $5(50 \%)$ & 10 \\
\hline & Ciprofloxacina & $7(58,3 \%)$ & - & $5(41,7 \%)$ & 12 \\
\hline & Cotrimoxazol & $7(63,6 \%)$ & - & $4(36,4 \%)$ & 11 \\
\hline & Ertapenem & $6(75 \%)$ & - & $2(25 \%)$ & 8 \\
\hline & Fosfomicina & $3(37,5 \%)$ & - & $5(62,5 \%)$ & 8 \\
\hline & Gentamicina & $8(66,7 \%)$ & - & $4(33,3 \%)$ & 12 \\
\hline & Nitrofurantoina & $6(75 \%)$ & - & $2(25 \%)$ & 8 \\
\hline & Norfloxacina & $3(37,5 \%)$ & - & $5(62,5 \%)$ & 8 \\
\hline & Ampicilina & 4 (40\%) & - & $6(60 \%)$ & 10 \\
\hline \multirow{6}{*}{$\begin{array}{l}\text { E. faecium } \\
(n=11)\end{array}$} & Daptomicina & $5(100 \%)$ & - & - & 5 \\
\hline & Levofloxacino & $3(37,5 \%)$ & - & $5(62,5 \%)$ & 8 \\
\hline & Linezolid & $9(100 \%)$ & - & - & 9 \\
\hline & Nitrofurantoina & $4(100 \%)$ & - & - & 4 \\
\hline & Vancomicina & $10(100 \%)$ & - & - & 10 \\
\hline & Ampicilina & $6(100 \%)$ & - & - & 6 \\
\hline \multirow{3}{*}{$\begin{array}{l}\text { E. faecalis } \\
(n=6)\end{array}$} & Levofloxacino & $4(66,7 \%)$ & - & $2(33,3 \%)$ & 6 \\
\hline & Nitrofurantoina & $3(100 \%)$ & - & - & 3 \\
\hline & Vancomicina & 5 (100\%) & - & - & 5 \\
\hline
\end{tabular}

*Número de aislados con resultados de antibiograma para cada antibiótico descrito. 
frecuentemente prescrito. Finalmente, un $0,5 \%$ de los pacientes fallecieron en urgencias y el $23,3 \%$ fueron hospitalizados $^{(3)}$.

En este sentido, comparando nuestros resultados con este análisis epidemiológico observamos un aumento en la prevalencia de las infecciones, con un perfil de pacientes de mayor edad y por tanto con mayor porcentaje de comorbilidades. Si bien tenemos que considerar una diferencia fundamental: en nuestro estudio el $36 \%$ de los ingresos se deben a una causa infecciosa y en el de SEMES el $14 \%$ de los atendidos en el SUH se deben a causa infecciosa, pero sólo el 23\% requieren ingreso. Por lo tanto, en nuestro estudio encontramos pacientes con infecciones más graves.

La asociación significativa encontrada entre las distintas comorbilidades y las infecciones sitúa a las primeras como factores de riesgo a tener en cuenta. Como parece evidente, la EPOC predispone a padecer infecciones respiratorias. Asimismo, padecer una uropatía o ser portador de catéteres predispone a padecer infecciones urinarias. Por otra parte, ingresar en estado de sepsis también debe considerarse un factor de riesgo importante, puesto que se asoció significativamente al aumento de mortalidad.

Las infecciones respiratorias fueron las predominantes, previsiblemente por la época del año en la que se recogieron los datos (invierno). El microorganismo mayoritariamente aislado fue $H$. influenzae, uno de los patógenos más prevalentes en las neumonías adquiridas en la comunidad y muy asociado a pacientes que padecen $\mathrm{EPOC}^{(13)}$, los cuales representan un alto porcentaje en nuestro grupo de estudio. Las infecciones urinarias fueron las segundas infecciones más prevalentes. Este hecho no sorprende puesto que son unas de las infecciones bacterianas más comunes en todos los estudios ${ }^{(3,6)}$. El microorganismo mayoritariamente aislado fue $E$. coli, responsable de la mitad de las infecciones urinarias con cultivo positivo de nuestro estudio.
Las pruebas microbiológicas no se llevaron a cabo en el $12,5 \%$ de los pacientes. Es una cifra considerablemente baja si lo comparamos con el estudio INFURG-SEMES $(56,7 \%)$. En cuanto a las causas de la falta de muestra esto puede ocurrir por diversas razones. En el caso de los urocultivos, estos a veces no se recogen si el paciente ha tomado antibiótico previo. Cuando la muestra a tomar es un hemocultivo, estos no se suelen recoger si el paciente no muestra signos febriles. Además, si la muestra a tomar ha de ser un esputo, a veces se complica la recogida si el paciente no es capaz de expectorar correctamente. Entre los pacientes en lo que las pruebas sí se llevaron a cabo, la más realizada fue el urocultivo, siendo este también el más rentable puesto que fue en el que se encontró un mayor porcentaje (64\%) de cultivos positivos para algún microorganismo.

Comparando los resultados de sensibilidad de los aislados de nuestro estudio con los datos proporcionados por el $\mathrm{ECDC}^{(14)}$ para los microorganismos más prevalentes, comprobamos que los datos obtenidos siguen en cierta medida el patrón esperado, a diferencia de $K$. pneumoniae que presenta una mayor proporción de cepas portadoras de BLEE ( $\beta$-lactamasas de espectro extendido) $(50 \%$ frente a $18 \%)$, carbapenemasas $(30 \%$ frente a $2 \%$ ) y resistencia a fluoroquinolonas (50\% frente a $18 \%$ ).

En el Estudio INFURG-SEMES, el registro de antibióticos prescritos refleja un predominio de la utilización de $\beta$-lactámicos $(45,5 \%)$ y fluoroquinolonas $(23,2 \%)^{(3)}$. En nuestro estudio la proporción es similar, $51,2 \%$ y $25,1 \%$, respectivamente. El elevado porcentaje de prescripciones empíricas evaluables que fueron acertadas nos lleva a la conclusión de que de forma general la prescripción del tratamiento empírico administrado en urgencias es correcta.

Con respecto a la mortalidad, observamos que el 7,3\% (edad media 82,1 años) de los pacientes fallecieron, bien por la infección que padecían o por las complicaciones de esta sobre su enfermedad de base. Esta tasa es

Tabla 6. Número de exitus y estancia media en el hospital según el tipo de infección.

\begin{tabular}{|c|c|c|}
\hline $\begin{array}{l}\text { Tipo de infección } \\
\text { (no de casos) }\end{array}$ & $\begin{array}{l}\text { Número de exitus } \\
\mathrm{n}(\%)\end{array}$ & $\begin{array}{l}\text { Estancia media en el hospital } \\
\text { en días } \pm D E\end{array}$ \\
\hline Respiratoria $(n=159)$ & $9(5,7)$ & $9,8 \pm 9$ \\
\hline Urinaria $(n=64)$ & $5(7,8)$ & $9,3 \pm 7,5$ \\
\hline Intraabdominal $(n=61)$ & $3(4,9)$ & $8 \pm 6,2$ \\
\hline Infecciones de piel y partes blandas $(n=12)$ & 0 & $13,8 \pm 14,9$ \\
\hline Meningitis ( $n=3$ ) & 0 & $20,7 \pm 11,5$ \\
\hline Mixta $(n=10)$ & $2(20)$ & $11,6 \pm 6,3$ \\
\hline Otras $(n=28)$ & $5(17,9)$ & $8,9 \pm 7,2$ \\
\hline Total $(\mathrm{N}=337)$ & $24(7,3)$ & $9,6 \pm 8,4$ \\
\hline
\end{tabular}


ligeramente superior a la encontrada en un estudio que analiza la mortalidad intrahospitalaria debida a causa infeciosa en el Servicio de Medicina Interna del Hospital Universitario Rio Hortega (5,8\%, edad media de 85,7 años) ${ }^{(15)}$.

Este estudio aporta datos de interés epidemiológico y de relevancia clínica para la práctica habitual y la elección de tratamiento antimicrobiano empírico. Es el primer estudio en indicar cuantos de los ingresos que se producen a través del Servicio de Urgencias tienen una causa infecciosa. Sin embargo, presenta ciertas limitaciones tales como un reducido tamaño muestral, naturaleza unicéntrica y diseño retrospectivo, por lo que estos resultados deberían ser confirmados en otros estudios.

En conclusión, es importante destacar que los procesos infecciosos suponen una elevada labor asistencial para el Servicio de Urgencias, así como una de las causas más frecuentes de ingreso hospitalario. Asimismo, se confirma que tanto las infecciones respiratorias como las urinarias e intraabdominales son las más frecuentes en nuestro entorno. La edad avanzada es uno de los principales factores de riesgo de morbimortalidad.

\section{Bibliografía}

[1] Pinner RW, Teutsch SM, Simonsen L, Klug LA, Graber $\mathrm{JM}$, Clarke MJ, et al. Trends in infectious diseases mortality in the United States. JAMA. 1996 Jan 17;275(3):189-93.

[2] Grupo de estudio de las infecciones en urgencias. Estudio epidemiológico de las infecciones en el área de urgencias. Emergencias, 2000; 12: 80-89.

[3] Martínez Ortiz de Zárate M, González Del Castillo J, Julián Jiménez A, Piñera Salmerón P, Llopis Roca F, Guardiola Tey JM and cols. Estudio INFURG-SEMES: epidemiología de las infecciones atendidas en los servicios de urgencias hospitalarios y evolución durante la última década. Emergencias, 2013; 25: 368-378.

[4] Geary U, Kennedy U. Toma de decisiones clínicas en Medicina de Urgencias y Emergencias. Emergencias. 2010;22:56-60.

[5] González-Castillo J, Candel FJ, Julián-Jiménez A. Antibiotics and timing in infectious disease in the emergency department. Enferm Infecc Microbiol Clin. 2013 Mar;31(3):173-80.

[6] Blanquer J, Solé-Violán J, Carvajal J, Lucena F. Infecciones comunitarias que requieren ingreso en UCI. Med Intensiva 2010 Sep;34(6):388-96.

[7] Siegel JD, Rhinehart E, Jackson M, Chiarello L, Healthcare Infection Control Practices Advisory Com- mittee. Management of multidrug-resistant organisms in health care settings, 2006. Am J Infect Control. 2007 Dec;35(10 Suppl 2):S165-193.

[8] López-Pueyo MJ, Barcenilla-Gaite F, Amaya-Villar R, Garnacho-Montero J. [Antibiotic multire-sistance in critical care units]. Med Intensiva. 2011 Feb;35(1):41-53.

[9] Martínez Ortíz de Zárate M. Aspectos epidemiológicos de las infecciones en las Áreas de Urgencias. Emergencias, 2001; 13, 44-50.

[10] Guerrero Espejo A, Tomás Dols S. Ingresos hospitalarios por enfermedades infecciosas: incidencia desde 1999 hasta 2003 en área sanitaria de la Comunidad Valenciana. Rev Esp Salud Pública. 2007 Aug;81(4):411-20.

[11] Gavazzi G, Krause K-H. Ageing and infection. Lancet Infect Dis. 2002 Nov;2(11):659-66.

[12] Kline KA, Bowdish DM. Infection in an aging population. Curr Opin Microbiol. 2016 Feb;29:63-7.

[13] Pletz MW, Rohde GG, Welte T, Kolditz M, Ott S. Advances in the prevention, management, and treatment of community-acquired pneumonia. F1000Research. 2016;5.

[14] ECDC: European Centre for Disease Prevention and Control. EARS-net. Disponible en: http://ecdc.europa.eu/en/activities/surveillance/ears-net/Pages /index.aspx (Consultado el 01/05/2016)

[15] Briongos-Figuero LS, Hernanz-Román L, Pineda-Alonso M, Vega-Tejedor G, Gómez-Traveso T, Sañudo-García S, et al. Inhospital mortality due to infectious disease in an Internal Medicine Department. Epidemiology and risk factors. Eur Rev Med Pharmacol Sci. 2015 Feb;19(4):567-72. 